\title{
Experimental Investigation of Electromagnetic Reverberation Characteristics as a function of UWB Frequencies
}

\author{
Aliou Bamba*1, Maria-Teresa Martinez-Ingles ${ }^{2}$, Davy P. Gaillot ${ }^{3}$, Emmeric Tanghe ${ }^{1}$ member IEEE \\ Brecht Hanssens ${ }^{1}$, Jose-Maria Molina-Garcia-Pardo ${ }^{2}$, Martine Lienard ${ }^{3}$ \\ Luc Martens ${ }^{1}$ member IEEE, and Wout Joseph ${ }^{1}$ senior member IEEE \\ ${ }^{1}$ Ghent University / iMinds - Department of Information Technology \\ Gaston Crommenlaan 8 box 201, B-9050 Ghent-Belgium \\ *Email: aliou.bamba@intec.ugent.be \\ ${ }^{2}$ Universidad Politecnica de Cartagena, Cartagena 30202, Spain \\ ${ }^{3}$ TELICE Group, IEMN, University of Lille \\ Bât. P3, 59655 Villeneuve d'Ascq, France
}

\begin{abstract}
The electromagnetic reverberation time characteristics of indoor environments are experimentally investigated from 2 to $10 \mathrm{GHz}$ with bandwidths up to $900 \mathrm{MHz}$. At a given frequency, the reverberation time is observed to be approximately constant up to 900 MHz. Moreover, the reverberation time decreases for increasing frequencies. Based on the theory of electromagnetic fields in cavities, a model to predict the room quality factor, reverberation time value, and average absorption coefficient is developed for the first time in indoor environments for the investigated frequency range. The validity and robustness of the model is investigated with data obtained for various environments, central frequencies, and bandwidths. The model is applied to another room over the whole $2-10 \mathrm{GHz}$ frequency band and a maximum and average relative error of $22.30 \%$ and $8.80 \%$ were obtained, respectively, with an rms error of $1.90 \mathrm{~ns}$. Furthermore, good agreement is obtained with measurements reported in the literature with settings falling into the model range; scenarios for which relative errors smaller than $10 \%$ were computed. The results demonstrate that this approach is not only an accurate alternative to the reverberation time measurements and computations of indoor environments in the 2-10 GHz frequency range but also a viable route to link propagation mechanisms in indoor scenarios with reverberation chambers.
\end{abstract}

Keywords: Reverberation time, modeling, dense multipath components, power density, Ultra-Wideband.

\section{INTRODUCTION}

$\mathrm{R}$ ECENT studies such as [1] have shown the importance of the Dense Multipath Components (DMC) in realistic indoor environments in terms of its contribution to the total power density. The DMC is usually considered as the remainder of the measurement data after removing all possible specular paths and includes the diffuse scattered fields plus weak specular components. The diffuse power density may represent up to $95 \%$ of the total power [1] in indoor environments and can be analytically expressed as a function of its reverberation time [2], [3]. This parameter, which characterizes the decay rate of the diffuse fields, is derived from the room electromagnetics theory [2], [4] and electromagnetic fields theory in cavities [5]. Hence, the reverberation time is the most important parameter regarding the diffuse absorption [2] and can be used, as an example, to predict the average power level in a room [6], [7]. The reverberation time has previously been investigated in office environments at a single frequency, i.e., 1.4, 2.3, 3.0, or 5.2 GHz [2][4], [7], [8]. However, the frequency dependence of the reverberation time or its characterization at higher frequencies have yet to be adressed to the best of the authors' knowledge. Currently, there are two approaches to determine the reverberation time in indoor environments, i.e., the measurement-based [3] and the computationalbased methods such as the radiosity [9]. The former solution is timeconsuming and difficult because it requires extensive measurement campaigns and post-processing steps, whereas the latter solution demands excessive time and memory resources, mainly at higher frequencies.

In contrast with indoor scenarios, the quality factor $(Q)$ is more frequently used to describe the capacity of reverberation chambers to store electromagnetic energy. Several works [10]-[13] have addressed the determination of $Q$ in reverberation chambers. Basically, $Q$ can be obtained either from the power-ratio method (frequency domain) or the decay-time method (time domain). The power-ratio method is based on the received power of a receiving antenna located in the reverberation chamber whereas the decay-time method is based on the reverberation time of the considered environment. The decay-time measurement was reported to yield a better estimate of $Q$ compared to the power-ratio method [5], [10], [13].

The novelty of this paper relies on the development of a frequencydependent model for $Q$ and the reverberation time for indoor scenarios, which can be considered as low- $Q$ reverberation rooms. This aspect is clearly missing in the literature and this work aims at filling this gap. Furthermore, the proposed approach links the absorption properties in indoor scenarios, characterized by the average absorption coefficient independent of the room dimensions, with the power losses in reverberation chambers, characterized by $Q$. This approach is motivated by the fact that similar mechanisms should be observed in both environments but at different scales due to the electromagnetic complexity of each scenario. Finally, this approach aims to provide an alternative to both measurements and computational methods.

The paper is organized as follows: $Q$ and the reverberation time approach from which the model is based on are presented in section II. The measurement scenarios and the methodology are also described. Section III presents the reverberation time dependency to the bandwidth as well as the frequency-dependent model for $Q$, the reverberation time, and average absorption coefficient. The validity of the reverberation time model is discussed in section IV. Finally, conclusions are drawn in section $\mathrm{V}$.

\section{Model, Scenarios, And Methods}

\section{A. Model for the $Q$-factor and reverberation time}

1) Frequency domain: From the electromagnetic fields theory in cavities, the quality factor of a resonant environment requires that 
four types of losses are accounted for. $Q$ is expressed as follows [5]:

$$
Q^{-1}=Q_{1}^{-1}+Q_{2}^{-1}+Q_{3}^{-1}+Q_{4}^{-1}
$$

where $Q_{1}, Q_{2}, Q_{3}$, and $Q_{4}$ are the quality factors due to the dissipated power in the walls $\left(\mathrm{P}_{1}\right)$, absorbed power in loading objects in the room $\left(\mathrm{P}_{2}\right)$, power lost in apertures $\left(\mathrm{P}_{3}\right)$, and dissipated power in the loads of receiving antennas $\left(\mathrm{P}_{4}\right)$, respectively.

The four quality factors exhibit frequency dependent behavior and are given by [5]:

$$
\begin{aligned}
& Q_{1}=\frac{\omega U}{P_{1}}=h_{1}(f), \quad Q_{2}=\frac{\omega U}{P_{2}}=h_{2}(f), \\
& Q_{3}=\frac{\omega U}{P_{3}}=h_{3}(f), \quad Q_{4}=\frac{\omega U}{P_{4}}=h_{4}\left(f^{3}\right), \quad \text { with } \omega=2 \pi \mathrm{f},
\end{aligned}
$$

where $U$, and $f$, are the energy in the room, and the considered frequency, respectively.

Given the expressions in (2), $Q$ is a cubic function with frequency as reported in [5], [10]. In particular, $Q_{1}, Q_{2}$, and $Q_{3}$ values are smaller compared to $Q_{4}$ at low frequencies such that their contribution to $Q$ is greater at high frequencies [5]. However, in contrast with reverberation chambers, $Q_{1}, Q_{2}$, and $Q_{3}$ are expected to be smaller in indoor environments since the power losses in the walls, objects, and apertures are larger.

2) Time domain: In the time domain, $Q$ is alternatively expressed as [5], [10], [13]:

$$
Q=2 \pi f \tau
$$

where $\tau$ is the room reverberation time given by Sabine's law [4]:

$$
\tau=\frac{4 V}{c_{0} \eta_{\text {avg }} A} .
$$

$\mathrm{V}, \mathrm{c}_{0}, \eta_{\text {avg }}$, and $\mathrm{A}$ are the room volume, light velocity, average fraction of energy absorbed by the surfaces, and room total area (including the floor, walls, ceiling, and objects).

Substituting (4) in (3), $Q$ becomes:

$$
Q=\frac{8 \pi f}{c_{0} \eta_{\text {avg }}} \times \frac{V}{A}=Q_{\text {density }} \times \frac{V}{A} .
$$

It is observed that $Q$ is proportional to V/A in agreement with [14], [15]. This also implies that a $Q$-factor density $Q_{\text {density }}\left[\mathrm{m}^{-1}\right]$ independent of the room dimensions can be introduced. $Q_{\text {density }}$ is the quality factor per volume per area and is expressed as follows:

$$
Q_{\text {density }}=\frac{8 \pi f}{c_{0} \eta_{\text {avg }}}=2 \pi f \tau \times \frac{A}{V} .
$$

Consequently, the frequency dependence of the reverberation time is given by :

$$
\tau(f)=\frac{Q_{\text {density }}}{2 \pi f} \times \frac{V}{A} .
$$

\section{B. Scenarios}

The measurements have been carried out in two laboratories located in the Universidad Politecnica de Cartagena, Spain. The laboratories are furnished by several closets, desktops, computers, shelves, etc. The first (resp. second) laboratory size is approximately $4.5 \mathrm{~m} \times 7 \mathrm{~m} \times 3 \mathrm{~m}$ (resp. $4.9 \mathrm{~m} \times 8.8 \mathrm{~m} \times 4.1 \mathrm{~m}$ ) resulting in a volume of $\sim 94.5 \mathrm{~m}^{3}$ (resp. $\sim 169 \mathrm{~m}^{3}$ ). The measurements performed in laboratory 1 (Fig. 1a) are used to characterize $Q$ and the reverberation time, as well as for modeling purposes, whereas the data from laboratory 2 (Fig. 1b) are used for the validation of the developed model (see section IV). The measurement scenarios and the channel sounder settings can be found in [16] but the main settings are recalled here for the reader: $i$ ) the frequency is ranging

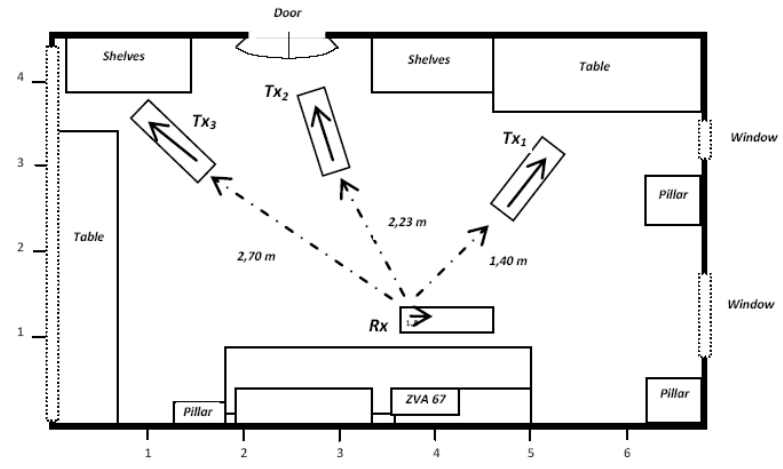

(a)

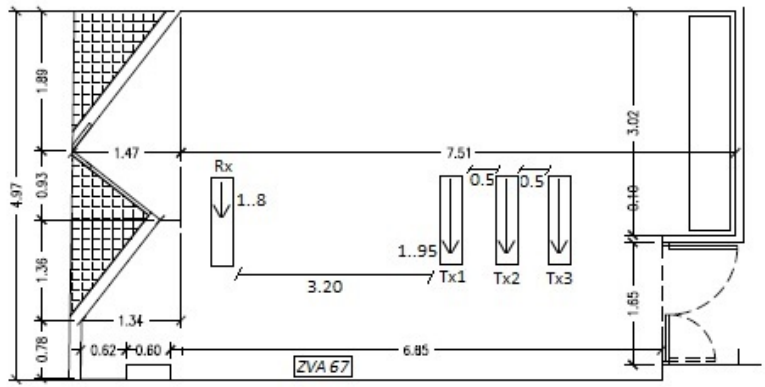

(b)

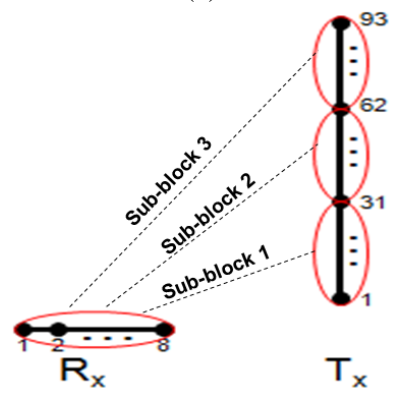

(c)

Fig. 1. (a) Laboratory $1\left(V=94.5 \mathrm{~m}^{3}\right.$ and $\left.A=132 \mathrm{~m}^{2}\right)$. The measurements are used to develop the $Q$-factor and reverberation time model. (b) Laboratory 2 $\left(V=169 \mathrm{~m}^{3}\right.$ and $A=195 \mathrm{~m}^{2}$ ). The measurements are used to validate the developed model. (c) Tx-Rx sub-blocks.

between 2 and $10 \mathrm{GHz}$ with 2048 frequency points and ii) three transmitter (Tx) blocks were considered. Each block consists of 95 Tx positions along a linear segment of $95 \mathrm{~cm}$, whereas the receiver $(\mathrm{Rx})$ occupied 8 positions along a linear segment of $70 \mathrm{~cm}$ (see Fig. 1c).

\section{Methods}

Each block (or segment of $95 \mathrm{~cm}$ ) is divided into 3 sub-blocks with 31 successive elements at the Tx side (in each sub-block) as shown in Fig. 1c (the $8 \mathrm{Rx}$ positions are retained). The Tx and Rx have been re-arranged so that the spatial correlation between two received signals in different sub-blocks is lower than 0.5 for the whole frequency band. This criterion is set to obtain spatially uncorrelated signals. Then, an averaged impulse response per sub-block is obtained with 8 positions of the Rx and 31 positions of the Tx (248 impulse responses). This is sufficiently large to remove the small-scale fading effects. The reverberation time is then experimentally determined from the averaged Power Delay Profile (PDP) as follows [3]:

$$
\tau=-\frac{10 \log (e)}{\text { slope }},
$$

where $e$ is Euler's number and slope is the slope of the PDP's linear tail. An example of the PDP is shown in Fig. 2. The reverberation time obtained from (4) is repeated for all sub-blocks. Finally, a single 


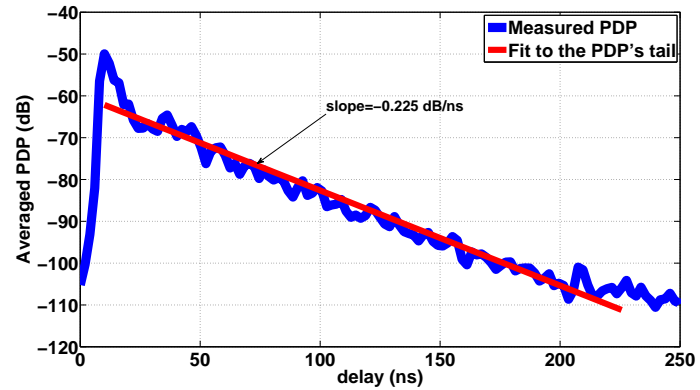

Fig. 2. A PDP averaged over all sub-blocks (in $\mathrm{dB}$ ) in laboratory 1.

value of the reverberation time - characteristic of the room regarding the diffuse absorption - is obtained by averaging the reverberation time values over all sub-blocks.

\section{RESULTS}

\section{A. Reverberation time as a function of bandwidth for laboratory 1}

Frequencies ranging from 2.5 to $9.5 \mathrm{GHz}$ with a step of $500 \mathrm{MHz}$ are investigated. The bandwidth varies from $100 \mathrm{MHz}$ up to $900 \mathrm{MHz}$ with a step of $50 \mathrm{MHz}$. Figure 3 presents the reverberation time as

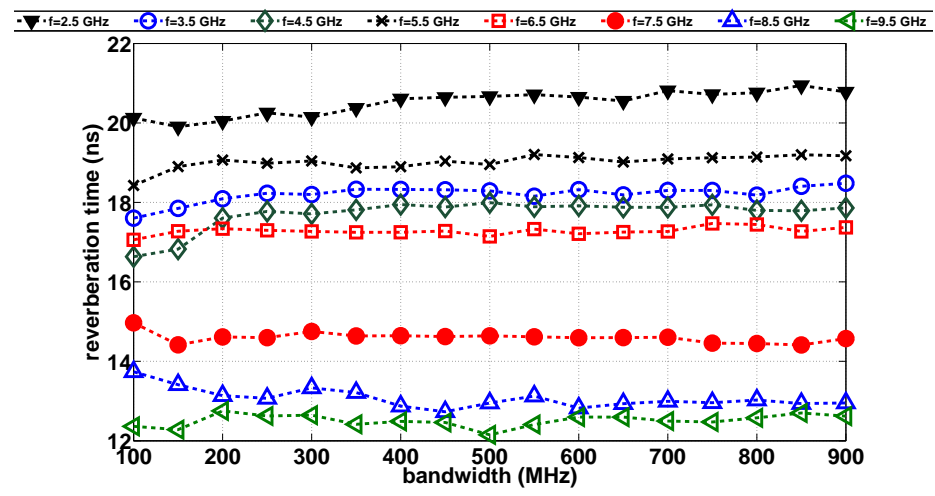

Fig. 3. Reverberation time as a function of bandwidth from 2.5 to $9.5 \mathrm{GHz}$ (laboratory 1).

a function of the bandwidth for different frequencies in laboratory 1. Only selected frequencies (legend of Fig. 3) are shown for the sake of clarity. The maximum relative standard deviation is about $2.2 \%$. This insignificant relative deviation clearly indicates that the reverberation time can be considered as constant over bands up to $900 \mathrm{MHz}$. This is probably because the building materials (bricks, concrete, limestone) properties do not vary significantly even over a wide frequency range [17]. A bandwidth of $500 \mathrm{MHz}$ is now considered for the rest of this study.

\section{B. $Q_{\text {density }}$ and reverberation time model}

At each frequency, the reverberation time values obtained for the 9 locations in laboratory 1 are averaged and used in (6) to obtain $Q_{\text {density. }}$. Figure 4 presents the experimental $Q_{\text {density }}$ and cubic polynomial fit as a function of frequency. The model choice is motivated by the fact that $Q$ is a cubic function with frequency as discussed previously in section II-A1. For the $2-10 \mathrm{GHz}$ range, the $Q_{\text {density }}$ model is given by the following equation:

$$
Q_{\text {density }}(f)=0.473 f^{3}-24.9 f^{2}+321 f-254, \text { with } \mathrm{R}^{2}=0.96
$$

where $0<f<10$ is the frequency in $\mathrm{GHz}$, and $\mathrm{R}^{2}$ is the goodness of fit. The reverberation time is obtained by substituting (9) into (7):

$$
\tau(f)=\frac{V\left(0.473 f^{3}-24.9 f^{2}+321 f-254\right)}{2 \pi f A},
$$

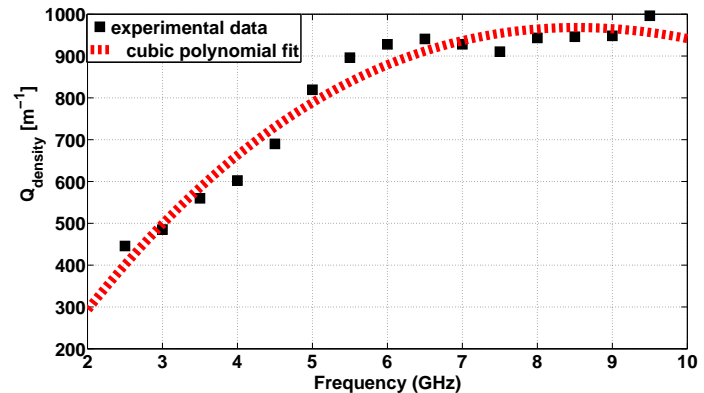

Fig. 4. Experimental (black squares) and model (red line) of $Q_{\text {density }}$ as a function of frequency for the laboratory 1 . A $500 \mathrm{MHz}$ bandwidth was considered and the $\mathrm{X}$-axis data represent the central frequency.

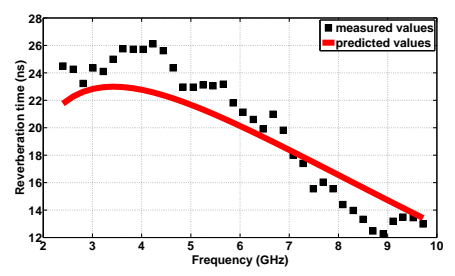

(a)

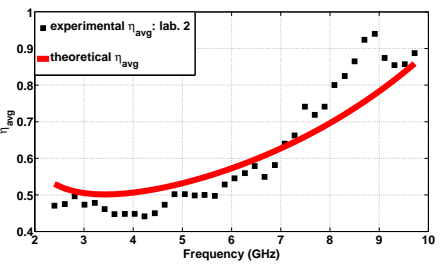

(b)
Fig. 5. Experimental (black squares) and model (red line) of $\tau$ (a) and $\eta_{\text {avg }}$ (b) in the laboratory 2 from 2-10 GHz. A $500 \mathrm{MHz}$ bandwidth was considered and the $\mathrm{x}$-axis data represents the central frequency.

where $\tau(\mathrm{f}), \mathrm{V}, \mathrm{A}$, and $\mathrm{f}$ are in nanoseconds, $\mathrm{m}^{3}, \mathrm{~m}^{2}$, and $\mathrm{GHz}$, respectively.

Finally, $\eta_{\text {avg }}$ can be obtained either from (4) or (5) and is shown to be, like $Q_{\text {density }}$, independent of the room dimensions:

$$
\eta_{\text {avg }}(f)=\frac{8 \pi}{c_{0}\left(0.473 f^{2}-24.9 f+321-254 f^{-1}\right)} .
$$

The independence of $\eta_{\text {avg }}$ of the environment is justified since it characterizes the absorption properties of the building materials and most of the buildings use the same materials.

\section{VALIDATION OF THE MODEL}

In this section, the validity of the model is discussed by applying (10) to different environments. For the sake of comparison, it is applied to an other room measured in this work over the whole frequency range of the model. In addition, it is also applied to experimental data reported in the literature from various research groups. These data were measured at frequencies and bandwidths for which the model can be used.

\section{A. Validation with Laboratory 2}

First, the proposed model is applied to measurements performed in laboratory 2 (Fig. 1b) over the complete $2-10 \mathrm{GHz}$ range. The experimental (black squares) and predicted (red curve) reverberation time values for laboratory 2 are presented from 2 to $10 \mathrm{GHz}$ in Fig. 5a. A maximum (resp. average) relative errors between the predicted and the measured values are about $22.30 \%$ (resp. $8.80 \%$ ) for the investigated frequency range. Moreover, the rms error is only about $1.90 \mathrm{~ns}$ for the complete frequency range. These low deviations show that the model accurately predicts the reverberation time values in the laboratory 2.

Figure 5a also shows the decrease of the reverberation time $\tau$ as the frequency increases. This indicates that the energy is fading faster away at higher frequencies compared to lower frequencies. This 
is confirmed by Fig. 5b which presents the frequency dependence of $\eta_{\text {avg. }}$. A maximum (resp. average) relative error between the predicted and the measured average absorption coefficient are of about $22.40 \%$ (resp. $8.70 \%$ ) for the investigated frequency range. When the frequency is low, the contribution of the dissipated power in the antenna loads to $Q$ cannot be neglected and becomes the major source of absorption in the environment; thus explaining the observed plateau. In contrast, as the frequency is increased, the losses from the building materials (walls, objects, and apertures) become larger and the reverberation time decreases. Indeed, the surface roughness plays a major role in this effect at higher frequencies; effect included in the reflection coefficient as follows [18], [19]:

$$
R_{\text {rough }}=\rho \times R_{\text {smooth }} .
$$

$\mathrm{R}_{\text {smooth }}$ is the Fresnel reflection coefficient for a smooth surface depending mainly on the surface dielectric properties [19], which in turn depends slightly of the frequency [17] and $\rho$ is the roughness attenuation factor. The roughness attenuation factor decreases as the frequency increases [18], [19]. Eventually, the reflection coefficient of rough surfaces decreases when the frequency increases. Therefore, the building materials of indoor environments (brick for the walls, limestone (or wood) for the floor, concrete, etc.) absorb more electromagnetic energy at higher frequencies (assume that their surfaces are rough), which in turn results in a decrease of the reverberation time as a function of the frequency as observed in Fig. 5a.

\section{B. Validation from literature}

1) Reference [4]: First, the model is compared to experimental data obtained in a rectangular room located in Aalborg University, Denmark $\left(V=522.5 \mathrm{~m}^{3}\right.$ and $\left.A=560^{2}\right)$ at $5.8 \mathrm{GHz}$ with $100 \mathrm{MHz}$ bandwidth. A reverberation time value of $\sim 22.10 \mathrm{~ns}$ was computed using (10) compared to the reported $24.10 \mathrm{~ns}$ resulting in a relative error of $9 \%$.

2) Reference [7]: Measurement-based reverberation time values of $18.40 \mathrm{~ns}$ and $16.70 \mathrm{~ns}$ were reported in two rooms $\mathrm{R} 4\left(\mathrm{~V}=74.4 \mathrm{~m}^{3}\right.$ and $\left.A=111 \mathrm{~m}^{2}\right)$ and $\mathrm{R} 3\left(V=55 \mathrm{~m}^{3}\right.$ and $\left.A=90 \mathrm{~m}^{2}\right)$ at $5.20 \mathrm{GHz}$ with $120 \mathrm{MHz}$ bandwidth, respectively. In comparison, $16.70 \mathrm{~ns}$ and $15.30 \mathrm{~ns}$ are computed with (10) yielding a relative error of only $9.20 \%$ and $8.30 \%$, respectively.

3) Reference [9]: Theoretical reverberation time values of $22.40 \mathrm{~ns}$ and $21.50 \mathrm{~ns}$ were obtained at $5.9 \mathrm{GHz}$ in the same room than [4] from radiosity (numerical simulation method), and theory, respectively. Our model predicts a reverberation time value of $21.60 \mathrm{~ns}$ at the same frequency. The relative error between the model and the simulations and theory are $3.60 \%$ and $0.50 \%$, respectively.

\section{Conclusions}

The frequency dependency of the electromagnetic $Q$-factor and reverberation time is experimentally investigated in indoor environments in the $2-10 \mathrm{GHz}$ frequency range. The results demonstrate that, for a given frequency, the reverberation time is constant over a large bandwidth - up to $900 \mathrm{MHz}$. In addition, the reverberation time decreases smoothly as the frequency is increased, indicating that the diffuse fields fade at a faster rate at higher frequencies. This phenomenon is attributed to the absorption coefficient frequency dependence of the building materials.

Based on the theory of electromagnetic fields in cavities, a model is proposed to predict the $Q$-factor, reverberation time, and average absorption coefficient over the investigated frequency range in indoor environments. It is concluded that the presented model not only agrees well with the reverberation time values reported in the literature but also with additional values measured in this work over the complete frequency range of $2-10 \mathrm{GHz}$. The validity and robustness of the model with data obtained for various environments, central frequencies, and frequency ranges demonstrate that this approach is an accurate alternative to the reverberation time measurements and computations in the frequency range of $2-10 \mathrm{GHz}$ in indoor environments. Future research consists in extending the model to the $\mathrm{mmW}$ range.

\section{ACKNOWLEDGMENT}

The research leading to these results has received funding from the European Union's Seventh Framework Programme (FP7/2007-2013) under grant agreement $\mathrm{n}^{\circ}$ 244149. This research has also received funding from the Fund for Scientific Research - Flanders (FWO-V, Belgium) project G.0325.11N. Emmeric Tanghe is a Post-Doctoral Fellow of the FWO-V (Research Foundation - Flanders).

\section{REFERENCES}

[1] J. Poutanen, J. Salmi, K. Haneda, V. M. Kolmonen, and P. Vainikainen. ”Angular and Shadowing Characteristics of Dense Multipath Components in Indoor Radio Channels". IEEE Transactions on Antennas and Propagation, 59: pages 245253, 2011.

[2] J. B. Andersen, K. L. Chee, M. Jacob, G. F. Pedersen, T. Kürner. "Reverberation and Absorption in an Aircraft Cabin with the Impact of Passengers". IEEE Trans. Antennas and Propag., 60 (5): pages 2472-2480, 2012.

[3] A. Bamba, W. Joseph, J. B. Andersen, E. Tanghe, G. Vermeeren, D. Plets, J. O. Nielsen, and L. Martens. "Experimental Assessment of Specific Absorption Rate Using Room Electromagnetics". IEEE Trans. Electromagn. Compat., 54 (4): pages 747-757, 2012.

[4] J. B. Andersen, J. Ø. Nielsen, G. F. Pedersen, G. Bauch, and M. Herdin. "Room Electromagnetics". IEEE Antennas Propag. Mag., 49, no. 2: pages 27-33, 2007.

[5] D. A. Hill. "Electromagnetic Fields in Cavities. Deterministic and Statistical Theories”. John Wiley \& Sons, Inc., 2009.

[6] C. L. Holloway, M. G. cotton, and P. McKenna. "A Model for Predicting the PoPower Delay Profile Characteristics Inside a Room”. IEEE Trans. Veh. Technol., 48 (4): pages 1110-1120, 1999.

[7] G. Steinbock, T. Pedersen, B. H. Fleury, W. Wang, and R. Raulefs. "Distance Dependent Model for the Delay Power Spectrum of In-room Channels". IEEE Trans. on Antennas and Propag., 61 (8): pages 4327-4340, 2013.

[8] J. Ø. Nielsen, J. B. Andersen, G. Frølund, M. Pelosi. "On Polarization and Frequency Dependence of Diffuse Indoor Propagation". In IEEE 74th Vehicular Technology Conference, San Francisco, USA, 2011.

[9] O. Franek, J. B. Andersen, G. F. Pedersen. "Diffuse Scattering Model of Indoor Wideband Propagation". IEEE Trans. Antennas Propag., 59 (8): pages 30063012, 2011.

[10] D. A. Hill, M. T. Ma, and A. R. Ondrejka. "Aperture Excitation of Electrically Large, Lossy Cavities". IEEE Trans. Electromagn. Compat., 36 (3): pages 169178, 1994.

[11] C. L. Holloway, D. A. Hill, J. M. Ladbury and G. Koepke. "Requirements for an Effective Reverberation Chamber: Unloaded or Load". IEEE Trans. Electromagn. Compat., 48 (1): pages 187-194, 2006.

[12] C. L. Holloway, D. A. Hill, J. M. Ladbury, P. F. Wilson, G. Koepke, and J. Coder. "On the Use of Reverberation Chambers to Simulate a Rician Radio Environment for the Testing of Wireless Devices". IEEE Trans. Antennas Propag., 54 (11): pages 3167-3177, 2006.

[13] V. Rajamani, C. F. Bunting, and J. C. West. "Differences in Quality Factor Estimation in Frequency and Time Domain". In Asia-Pacific Symposium on Electromagnetic Compatibility (APEMC), 2012.

[14] P. Corona, G. Latmiral, E. Paolini, and L. Piccioli. "Use of a Reverberating Enclosure for Measurements of Radiated Power in the Microwave Range". IEEE Trans. Electromagn. Compat., 18 (2): pages 54-59, 1976.

[15] U. S. Inan and A. S. Inan. "Electromagnetic Wave”. Prentice Hall, 2000.

[16] M. T. Martinez-Ingles, J. M. Molina-Garcia-Pardo, J. V. Rodriguez, J. PascualGarcia, and L. Juan-Llacer. "Experimental Comparison between Centimetre and Millimeter - Wave Ultra - Wideband Radio Channels". Radio Science, 49(6): pages 450-458, 2014.

[17] S. Stavrou and S. R. Saunders. "Review of constitutive parameters of building materials". In IEE twelfth International Conference on Antennas and Propagation (ICAP), University of Exeter, UK., 31 March-03 April 2003.

[18] F. Delfino, P. Girdinio, L. Minervini, M. Nervi. "Electromagnetic plane wave scattering from building surfaces". COMPEL - The international journal for computation and mathematics in electrical and electronic engineering, 25 (4): pages 1007-1018, 2006.

[19] R. Piesiewicz, C. Jansen, D. Mittleman, T. Kleine-Ostmann, M. Koch, and T. K"urner. "Scattering Analysis for the Modeling of $\mathrm{THz}$ Communication Systems". IEEE Trans. Antennas Propag., 55(11): pages 3002-3009, 2007. 